\title{
Reviewer Acknowledgements for Global Journal of Health Science, Vol. 9, No. 7
}

Global Journal of Health Science wishes to acknowledge the following individuals for their assistance with peer review of manuscripts for this issue. Their help and contributions in maintaining the quality of the journal are greatly appreciated.

Global Journal of Health Science is recruiting reviewers for the journal. If you are interested in becoming a reviewer, we welcome you to join us. Please find the application form and details at http://recruitment.ccsenet.org and e-mail the completed application form to gjhs@ccsenet.org.

\section{Reviewers for Volume 9, Number 7}

Aljameel Albandary,King Saud University, Saudi Arabia

Delfina Gabriela Ramos, Polytechnic Institute of Cávado and Ave, Barcelos, Portugal

Dharampal G. Dambhare, Mahatma Gandhi Institute of Medical Sciences, India

Dilek Pirim, Uludag University, Bursa, Turkey

Evangelia Mavrikaki, National \& Kapodistrian University of Athens, Greece

Evanthia Sakellari, Technological Educational Institute of Athens, Greece

Farahnaz Amini, UCSI University, Malaysia

Fernanda Lima-Christian, Federal University of Santa Catarina, Brazil

Gabriel Gulis, University of Southern Denmark, Denmark

Giovana Fernandes, Univ Estadual Paulista - UNESP, Brazil

Hui Peng, Yijishan Hospital of Wannan Medical College, China

Hülya YARDIMCI, Ankara University, Turkey

Jan Chrastina, Palacký University, Czech Republic

Jichuan Wang, The George Washington University, United States

Jose R Cordon, Universidad de Cadiz (Spain); SASEMAR(M. Fomento-Spain)-Spanish Goverment, Spain

Jose Manuel Hernandez-Padilla, Middlesex University, United Kingdom

José Joaquín Mira, Universidad Miguel Hernández, Spain

Kinley Wangdi, Australian National University, Australia

Lisa Scherer, University of Nebraska at Omaha, United States

Liye Suo, The Ohio State University, United States

Loray Daws, British Columbia Masterson Institute, Canada

Nant Thin Thin Hmwe, University Malaysia Sabah, Malaysia

Nazisa Hejazi, National University, Malaysia

Piacherski Valery, Department of Anesthesiology and Intensive Care of Mogilev Regional Hospital, Belarus

Randy Warren Beck, Murdoch University, Australia

Raúl Quevedo-Blasco, Universidad de Granada (CIMCYC-UGR), Spain

Regina E.Ella, University Of Calabar, Nigeria

Robert Sloan, Kagoshima University Graduate School of Medical and Dental Sciences, Japan

Satoshi Horiuchi, Iwate Prefectural University, Japan

Srikrishna Sulgodu Ramachandra, Public Health Foundation of India, India

Thammanard Charernboon, Thammasat University, Thailand

Thomas Leiblein,Zurich University of Applied Sciences, Switzerland

Trisha Dunning, Deakin University and Barwon Health, Australia

Tsan Yang, Meiho University, Taiwan, Province of China

Yan Ma, Beth Israel Deaconess Medical Center, Harvard Medical School, United States 